\title{
Mulheres profissionais do sexo: discurso sobre o uso do preservativo e sua autopercepção de vulnerabilidade ao HIV
}

\author{
Professional women of the sex: discourse on the use of the condom and its self-perception of HIV \\ vulnerability
}

Mujeres profesionales del sexo: discurso sobre el uso del condón y su auto percepción de vulnerabilidad al VIH

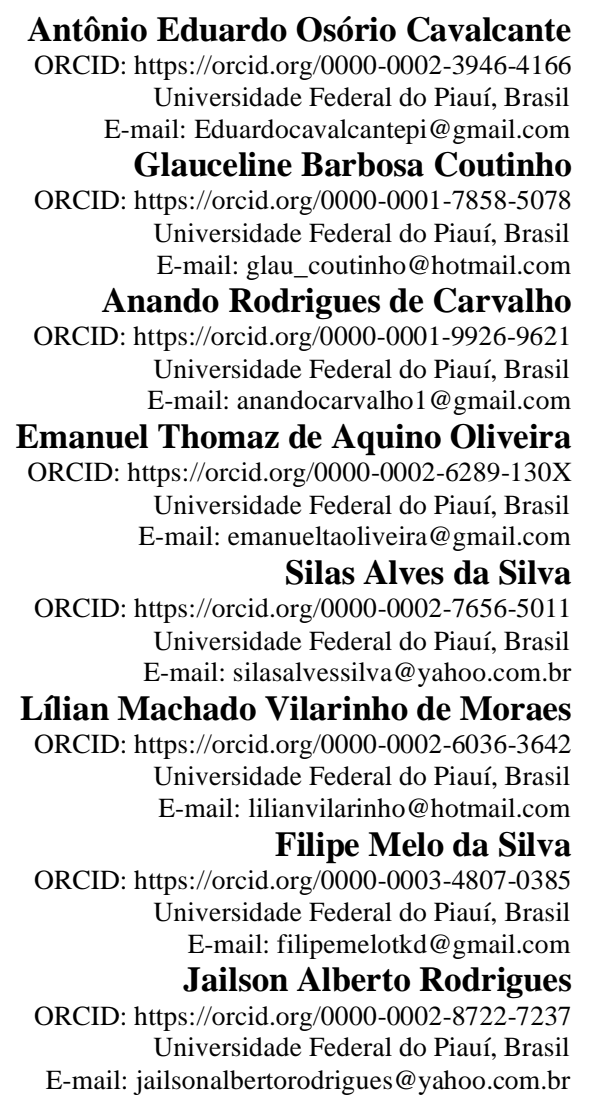

\begin{abstract}
Resumo
A síndrome da imunodeficiência adquirida - aids é o estágio mais avançado do complexo infectocontagioso do vírus da imunodeficiência humana - HIV. No Brasil, surgiram aproximadamente 40 mil novos casos de aids nos últimos cinco anos. Dentre os principais grupos acometidos estão as mulheres profissionais do sexo - MPS. Mediante esse fato, este estudo pretendeu apresentar o discurso coletivo das MPS sobre o uso do preservativo e, sua autopercepção de vulnerabilidade frente à infecção pelo HIV. Trata-se de uma pesquisa descritiva, transversal, analítica, de abordagem qualitativa, realizada com MPS em Floriano, Piauí. A coleta dos dados ocorreu entre os meses de setembro de 2019 a março de 2020. O discurso do sujeito coletivo - DSC proposto por Fiorin norteou a análise dos depoimentos. De maneira unânime $(n=5)$, as MPS referiram conhecer a necessidade do uso de preservativos na minimização da vulnerabilidade ao HIV durante todas as relações sexuais. Para elas, o uso da camisinha é um método essencial na prevenção à saúde, durante as relações sexuais. 80,0\% $(\mathrm{n}=4)$ das mulheres participantes referiram não se sentirem vulneráveis ao HIV. Ao investigar as percepções das MPS, no que tange ao uso do preservativo, foi identificada boa aceitação. Há associação do seu uso como uma questão de saúde, que está acima da oportunidade de ganhar dinheiro.
\end{abstract}

Palavras-chave: Profissionais do sexo; HIV; Vulnerabilidade social. 


\begin{abstract}
The Acquired immunodeficiency syndrome - AIDS is the most advanced stage of the contagious complex of human immunodeficiency virus - HIV infection. In Brazil, approximately 40 thousand new AIDS cases have appeared in the last five years. Among the main groups affected are women sex workers - MPS. Based on this fact, this study aimed to present the collective discourse of the MPS on condom use and their self-perception of vulnerability against of HIV infection. This is a descriptive, cross-sectional, analytical, qualitative study, carried out with MPS in Floriano, Piauí State. Data collection took place between the months of September 2019 and March 2020. The collective subject discourse method - DSC proposed by Fiorin guided the analysis of the participants' testimonies. All MPS ( $\mathrm{n}=5$ ) reported knowing the need to use condoms to minimize vulnerability to HIV during all sexual relations. For them, the use of condoms is an essential method to prevent health during sexual intercourse. $80.0 \%(n=4)$ of the participating women reported not feeling vulnerable to HIV. When investigating the perceptions of MPS about condom use by them during sex, good acceptance was identified among them. for them, the use of condoms is a health issue and is above the opportunity to earn money.
\end{abstract}

Keywords: Sex workers; HIV; Social vulnerability.

\title{
Resumen
}

El síndrome de inmunodeficiencia adquirida: el sida es la etapa más avanzada del complejo infeccioso y contagioso del virus de la inmunodeficiencia humana, el VIH. En Brasil, han aparecido aproximadamente 40 mil nuevos casos de SIDA en los últimos cinco años. Entre los principales grupos afectados se encuentran las trabajadoras sexuales - MPS. Ante este hecho, este estudio tuvo como objetivo presentar el discurso colectivo de los MPS sobre el uso del condón y su autopercepción de vulnerabilidad frente a la infección por VIH. Se trata de un estudio descriptivo, transversal, analítico con enfoque cualitativo, realizado con MPS en Floriano, Piauí. La recolección de datos tuvo lugar entre los meses de septiembre de 2019 y marzo de 2020. El discurso del sujeto colectivo - DSC propuesto por Fiorin guió el análisis de los enunciados. Unánimemente $(\mathrm{n}=5)$, el MPS informó conocer la necesidad de usar condones para minimizar la vulnerabilidad al VIH durante todas las relaciones sexuales. Para ellos, el uso de condones es un método fundamental para prevenir la salud durante las relaciones sexuales. El 80.0\% $(\mathrm{n}=4)$ de las mujeres participantes informaron no sentirse vulnerables al VIH. Al investigar las percepciones de MPS, con respecto al uso de condones, se identificó buena aceptación. Existe una asociación de su uso como problema de salud, que está por encima de la oportunidad de ganar dinero.

Palabras clave: trabajadoras sexuales; VIH; Vulnerabilidad social.

\section{Introdução}

A prática da prostituição trata-se de uma das atividades comerciais mais antiga da história da humanidade. Definida pelo exercício da prática sexual remunerada, não sendo requerida a existência de qualquer vínculo afetivo entre as pessoas que a realizam (Penha et al., 2015).

Desde a concepção dessa atividade, a imagem da prostituta sempre esteve ligada à promiscuidade, safadeza, imoralidade, impureza e libertinagem. Esta condição da noção de prostituição, estabelecida social e culturalmente, foi capaz de produzir uma farta gama de preconceitos, que assim como qualquer preconcepção diminui a pessoa como humana. O termo prostituição, deriva do latim prosto, que quer dizer 'estar às vistas, à espera de quem quer chegar ou estar exposto ao olhar público', 'é aquela que costura para fora', 'meteriz' [...] é a prática sexual remunerada habitual e promíscua (Ribeiro, 2017). Em sentido conotativo, o termo perpassa entre os mais variados significados, destacando-se os mais utilizados: 'expor algo publicamente', 'vender', 'praticar serviços sexuais em troca de dinheiro'. Na linguagem popular, a prostituição recebeu outras denominações, tais como: 'a mais antiga das profissões', 'vida fácil', 'programa' (Soares et al., 2015).

Ao se referir ao trabalho da profissional do sexo, a terminologia mais utilizada para tal, trata-se da conotação 'fazer programa'. Embora trate-se de uma prática milenar, somente no ano 2002 a categoria 'profissionais do sexo' foi incluída na Classificação Brasileira de Ocupações (CBO) do Ministério do Trabalho e Emprego (MTE) (Brasil, 2010).

Ao atentar-se para os motivos pelos quais as mulheres optam pela realização dessa atividade, não há motivos universais que a levem para essa escolha. Apesar da pluralidade dos motivos, diversos estudos, ao longo dos anos, conseguiram determinar alguns problemas sociais, que podem contribuir para o fenômeno social 'prostituição'. Pobreza, uso de substâncias ilícitas, estrutura familiar deficitária, abuso sexual são fatores que facilitam a entrada na prostituição (Penha et al., 2015). 
Assim, o sexo revela-se como uma mercadoria, sendo a fonte de renda dessas mulheres, caracterizando-as como um grupo que vive, marginalmente, aos riscos da profissão, dentre os quais citam-se as infecções sexualmente transmissíveis - IST, em especial, ao HIV/Aids (Cruz et al., 2016).

Esse grupo de mulheres está representado como uma população de alto risco para as infecções sexualmente transmissíveis, e em especial, ao HIV/Aids. Neste contexto, as mulheres profissionais do sexo - MPS tendem a fazer parte, diante da forma como a aids se apresenta, em termos epidemiológicos, dessa realidade. Principalmente por serem um grupo social que tem a atividade sexual como matéria prima do seu processo de trabalho. Além disso, muitas vezes esse ato sexual é realizado com ausência de preservativo, podendo ser advinda ou acentuar vulnerabilidades individuais, sociais e programáticas, tais como a baixa escolaridade, acesso restrito aos serviços de saúde, baixo poder de negociação do uso do preservativo com o parceiro e estigmas sociais (Patrício et al., 2018).

Portanto, para se compreender os determinantes de vulnerabilidade das MPS necessita-se traçar intervenções adequadas para a problemática envolvendo sua saúde. Fato esse que motivou a realização deste estudo, o qual pretendeu apresentar o discurso coletivo das MPS sobre o uso do preservativo e, sua autopercepção de vulnerabilidade frente à infecção pelo ao HIV.

\section{Metodologia}

Esta pesquisa é de natureza qualitativa, transversal e analítica, realizada com mulheres profissionais do sexo em todo o município de Floriano, Piauí, entre o período de setembro de 2019 a março de 2020. Entretanto, trata-se de um recorte de uma pesquisa quantiqualitativa mais ampla intitulada "Autopercepção da vulnerabilidade ao HIV entre mulheres profissionais do sexo do município de Floriano-PI", a mesma segue em andamento, com previsão de encerramento para julho de 2021. Uma vez que outros aspectos continuam em investigação.

Floriano é um município brasileiro do estado do Piauí, situado na Zona Fisiográfica do Médio Parnaíba, à margem direita desse mesmo Rio, em frente à cidade de Barão de Grajaú, Maranhão. A cidade fica a $240 \mathrm{~km}$ da capital do estado do Piauí.

No tocante à saúde, o município oferece amplo atendimento, seja na esfera pública ou privada, dispondo de um Hospital Regional de médio porte, vinte e quatro Unidades Básicas de Saúde e um Centro de Testagem e Aconselhamento em todo o território. Possui, ainda, uma rede de clínicas privadas com um hospital particular de pequeno porte. Estes serviços atendem à demanda da macrorregião em diversas especialidades.

No contexto das mulheres profissionais do sexo, embora seu exercício esteja dentro da legalidade, regulamentada e prevista na Classificação Brasileira de Ocupações - CBO, as Casas de Prostituição são proibidas no Brasil. Assim, não existem dados oficiais que descrevam a quantidade exata de casas noturnas no município. Entretanto, é possível que se encontrem mulheres no exercício de sua profissão em ruas e estabelecimentos por toda a cidade, assim, coube ao pesquisador, sua procura e realização da entrevista.

A realização do estudo deu-se ao passo em que houvera o encontro de locais de trabalho de mulheres profissionais do sexo, ou ainda, de MPS que trabalham individualmente, durante todos os períodos do dia, ou seja, pela manhã, tarde ou noite, havendo somente a necessidade de disponibilidade dessas profissionais e do pesquisador.

A amostra não probabilística foi definida por meio da técnica de amostragem "bola de neve", ou Snowball Sampling, (Biernacki \& Waldorf, 1981). Essa técnica baseia-se na utilização de cadeias de referência, caracterizada pela identificação dos sujeitos de interesse, a partir da população que já foi pesquisada. As participantes que finalizavam a pesquisa indicavam novas participantes, que por sua vez indicariam outras, de maneira sucessiva, até que se obtivesse uma amostra satisfatória, capaz de alcançar os objetivos propostos. 
Durante a coleta de dados foi solicitado às entrevistadas que indicassem outras pessoas com perfil participativo para serem entrevistadas. Desta maneira, a abordagem tornou-se facilitada. Foi solicitado e permitido o máximo de indicações, favorecendo maior probabilidade de sucesso na busca pelo público-alvo.

Um fator relevante a ser citado, trata-se de que as indicações dadas pelas participantes não garantiram que haveria aceitação por parte das participantes indicadas, nem revelaram à indicadora, que a pessoa indicada aceitou participar da pesquisa. Assim, essa técnica de amostragem não pode 'quebrar' o sigilo dos indivíduos participantes.

A técnica Snowball Sampling é de amostragem não probabilística, por isso, não permite que se determine a probabilidade de seleção de cada participante na amostra. Por outro lado, as cadeias de referência facilitam a identificação dos sujeitos de pesquisa (Colin \& Pelicione, 2018). Portanto, a amostra não probabilística foi definida por conveniência e casualidade, não sendo possível prever o tamanho amostral $(\mathrm{N})$ antes da realização da pesquisa. A amostra se deu por encerrada na medida em que se obteve saturação amostral, por meio do ponto de saturação das respostas, que se tornaram constantemente repetitivas.

Quando identificado um padrão constante nas respostas dadas, por saturação teórica - quando os dados obtidos começaram a demonstrar repetição ou redundância, o delineamento amostral fora encerrado. Em outras palavras, quando os dados obtidos não agregaram novas informações, esta amostra excedente deixou de ser necessária, já que não alteraria a compreensão do fenômeno estudado, pois trata-se de um critério que permite estabelecer a validade de um conjunto de dados (Nascimento et al., 2018).

Foram inseridas no presente estudo mulheres profissionais do sexo, maiores de 18 anos, que aceitaram, voluntariamente, participar da pesquisa mediante assinatura do Termo de Consentimento Livre e Esclarecido - TCLE. Fora realizada leitura, discussão e esclarecimento do TCLE com todas as participantes, além da leitura do instrumento norteador a ser aplicado, em caso de participantes analfabetas, visando a compreensão exata da finalidade do estudo, bem como os benefícios a eles associados.

Como critério de exclusão constaram as situações em que a participante possuísse menos de 18 anos e/ou não apresentasse condições cognitivas para responder ao instrumento norteador da pesquisa.

Os dados foram coletados por meio de um instrumento norteador elaborado pelos próprios pesquisadores, que possuía como objetivo a identificação da autopercepção de vulnerabilidade ao HIV entre as profissionais do sexo estudadas. A coleta de dados foi realizada em toda a área urbana do município de Floriano-PI.

Por não ser possível prever os locais no município em que pudessem se encontrar as MPS, uma vez que estas poderiam situar-se nos mais diversos locais distribuídos na cidade, coube ao pesquisador, a partir do método de Snowball, identificar as possíveis participantes, e dar prosseguimento à coleta dos dados.

A coleta dos dados compreendeu uma única etapa: a aplicação do instrumento norteador para avaliar a autopercepção de vulnerabilidade ao HIV. As questões variavam entre objetivas e subjetivas, contendo questões que permitiam a análise das características da prática sexual de MPS, no que concerne a utilização de métodos preventivos de infecção pelo HIV; a realização de testes anti-HIV e a motivação para realizá-los e a autopercepção da própria vulnerabilidade para o HIV.

Para a análise dos dados, o referencial qualitativo fora obtido por meio análise do discurso do sujeito coletivo proposta por Fiorin (1997), que compreendeu duas etapas, sendo elas a análise de conteúdo e análise do discurso. A primeira compreende a análise de conteúdo, onde se buscou compreender as falas, depoimentos e reproduzir textos. A segunda etapa, mostra o posicionamento coletivo dos sujeitos, a análise do discurso ou "voz social". Esta necessita de um maior grau de abstração de uma ideologia, por parte de quem analisa.

O estudo central foi submetido ao Comitê de Ética Em Pesquisa (CEP) da Universidade Federal do Piauí - UFPI, e, após apreciação e aprovação pelo parecer de $\mathrm{N}^{\circ}$ 4.035.629. A pesquisa foi efetivada, respeitando os princípios éticos previstos 
nas recomendações nacionais. Por isso, optou-se, por exemplo, em denominar as sujeitas participantes, por meio de pseudônimos.

\title{
3. Resultados e Discussão
}

A idade das participantes, cinco no total $(\mathrm{N}=5)$, variou de 22 a 29 anos, sendo a faixa de 18 a 25 anos a prevalente $(80,0 \% ; n=4)$. A maioria das mulheres declararam-se pardas $(80,0 \% ; n=4)$; católicas $(60,0 \% ; n=3)$; solteiras $(80,0 \% ; n=4)$, com rendimento de 1 a 3 salários-mínimos $(60,0 \% ; n=3)$. Ainda, com relação a escolaridade, $80,0 \%(n=4)$ concluíram o Ensino Fundamental, enquanto 20,0\% $(\mathrm{n}=1)$ concluíram o Ensino Médio, e 20,0\% ( $\mathrm{n}=1)$ o Ensino Superior. A renda, embora relatada na faixa de 1 a 3 salários-mínimos, 60,0\% delas relatou ser complementada com outro trabalho, enquanto $20,0 \%(\mathrm{n}=1)$ não possuíam outra fonte de renda, e outras $20,0 \%(\mathrm{n}=1)$ não responderam.

A idade se constitui como característica de grande influência na busca da clientela, uma vez que idades mais avançadas não cumprem os requisitos exigidos pelos clientes, ainda que possam ser consideradas mulheres com mais experiência (Soares et al., 2015).

A prevalência de profissionais do sexo de cor parda pode ser explicada pela maioria da população brasileira declararse parda, e esse fato pode contribuir para que, sob a ótica de um país miscigenado, ainda que essas pessoas pertençam, por características, a uma outra cor, estas, ainda assim, se reconheçam como pardas (Alves, De Jesus \& Diaz, 2017).

Quanto à religião, as que optaram por responder ao questionamento, declararam serem católicas, dado também obtido em estudo realizado em Fortaleza, Ceará, por Sousa e colaboradores (2017b), onde a maioria das MPS abordadas declararamse católicas, além disso, refere-se que esse fato pode ser justificado por se tratar da religião que reúne mais adeptos no mundo, com o Brasil a ser um dos países com a maior presença de cristãos católicos em relação à outras religiões.

Em relação à escolaridade, maior parte das mulheres não havia concluído o ensino médio. Todas as participantes haviam concluído, ao menos, o ensino fundamental incompleto, enquanto $20 \%$ concluíram o ensino médio, e outros $20,0 \%$ o ensino superior. Dados semelhantes foram obtidos em alguns estudos com profissionais do sexo (Martins et al., 2018).

A situação conjugal das mulheres encontrou amplo predomínio de mulheres solteiras (80,0\%), corroborando com outros estudos que visaram conhecer o estado civil dessas profissionais. Sobre sua renda mensal, vários fatores podem interferir na remuneração mensal das mulheres, entre eles: preço do atendimento, idade da mulher, quantidade de dias trabalhados, número de atendimentos realizados por dia, semanalmente ou mensalmente e práticas realizadas durante o ato sexual (Santos et al., 2019).

De maneira unânime ( $\mathrm{n}=5$ ), as MPS referiram conhecer a necessidade do uso de preservativos na minimização da vulnerabilidade ao HIV durante o sexo por via oral, vaginal e/ou anal. Quando questionadas quanto ao motivo da real necessidade da utilização, referiram:

\author{
'é fundamental' (MPS Moana). \\ 'serve pra se prevenir sempre' (MPS Aurora). \\ 'a saúde está em primeiro lugar e o dinheiro é só um complemento' (MPS Elsa).
}

De acordo com as mulheres abordadas, o uso da camisinha durante o ato sexual está diretamente ligado a uma questão de saúde, tanto no âmbito sexual quanto no reprodutivo, colocando-a, inclusive, acima do dinheiro pelo qual recebem ao prestar esse serviço. Embora não tenham detalhado sobre quais doenças, ou ainda, por qual motivo o uso da camisinha é fundamental, o conhecimento da sua necessidade pode configurar como fator de proteção, mesmo diante da falta de compreensão da alta complexidade envolvendo a saúde sexual e reprodutiva. 
Essa ideia, passada repetidas vezes através do discurso de campanhas e políticas de saúde pública, que visam incentivar a relação sexual segura, bem como a facilidade de se obter informações em circunstâncias onde o caminho à informação é tangível, sugere que essas mulheres reconheçam o seu uso, baseado no discurso em volta do estabelecimento da saúde como prioridade. Entretanto, é necessário que os profissionais de saúde fortaleçam o implemento de ações educativas que visem aperfeiçoar o conhecimento das mulheres acerca da importância da utilização dos preservativos, potencializando a adesão ao seu uso (Lima et al., 2016).

Apesar do exposto, conhecer a necessidade da utilização de um método de prevenção como o preservativo, ainda que justificada de acordo com as suas percepções, não implica dizer que o seu uso é de fato, realizado. Assim, corroborando com a ideia de que, com o aumento dos índices epidemiológicos das IST/HIV/Aids no Brasil, o desenvolvimento de campanhas educativas para conscientizar a população sobre os métodos de prevenção dessas afecções é uma das prioridades da saúde pública (Dourado et al., 2015).

Quanto ao perfil da autopercepção de vulnerabilidade ao HIV, 80,0\% $(\mathrm{n}=4)$ das mulheres pesquisadas referiram não se sentirem vulneráveis, enquanto $20,0 \%(\mathrm{n}=1)$ não responderam. Com base na resposta das participantes, foi solicitado que explicassem os motivos pelos quais sentiam-se ou não, vulneráveis ao HIV/aids, e aquelas que optaram pela resposta, mencionaram que não se sentiam, acrescentando as seguintes ideias:

'pois me previno de todas as maneiras cabiveis' (MPS Moana);

'porque uso preservativo sempre' (MPS Aurora).

Alguns pontos permeiam a análise de maneira conjunta, dentre eles, o significado pessoal atribuído ao conceito de vulnerabilidade. Para as pessoas, vulnerabilidade para IST/HIV/Aids remete a uma noção de exposição a situação de risco por diversos fatores, entre os quais citam-se a irresponsabilidade e a falta de cuidado (Costa et al., 2014). Além disso, o termo pode ser atrelado a uma noção de fragilidade muito específica, como a que ocorre a pessoas com condições delicadas, envolvendo diversos termos equivocados.

As medidas preventivas contra o HIV dão-se por meio da utilização do preservativo, principalmente. A percepção da vulnerabilidade pessoal e a conduta sexual, tendo em vista as diferentes dinâmicas da epidemia - 'heterossexualização', 'feminização' e 'pauperização' -, as quais guardam uma relação estreita com a heterogeneidade socioeconômica e cultural da população, geram diferentes níveis de vulnerabilidade no país (Miranda-Ribeiro et al., 2015).

A partir disso, buscou-se conhecer a percepção dessas mulheres acerca do uso do preservativo, uma vez que este é o método utilizado por elas e constitui-se como a principal forma de prevenção contra IST/HIV/Aids disponível e, a sua utilização é de fundamental importância para a manutenção do controle da epidemia no Brasil. Assim, averiguou-se os motivos de sua adesão ou rejeição, caso houvesse. Conforme expresso no Quadro 1. 
Quadro 1 - Percepção de mulheres profissionais do sexo sobre o uso de preservativos durante a relação sexual. Floriano-PI, 2020. $(\mathrm{n}=5)$.

\begin{tabular}{|l|l|l|}
\hline Pergunta: O que você acha do uso da camisinha? & Unidade ideológica & DSC \\
\hline 'Previne DST'- MPS Rapunzel & & \\
'Indispensável'- MPS Moana & & O uso da camisinha é um \\
'Necessário e essencial, previne doenças, gravidez e & & método essencial na \\
muitas outras coisas'-MPS Elsa & Prevenção primária da saúde & prevenção à saúde, durante as \\
'Essencial' - MPS Aurora & & relações sexuais. \\
'Necessário em todas as relações sexuais' - MPS & & \\
Jasmine & & \\
\hline
\end{tabular}

Fonte: Autores.

Observa-se na construção do discurso dos sujeitos a continuidade da propagação das informações que são distribuídas pelas políticas de saúde em relação aos preservativos. Menciona-se o fato de todas as falas retratarem uma construção socialmente aceita acerca do seu uso, colocando a saúde como prioridade e sua utilização como fundamental para a prática laboral segura.

Estudos realizados na América Latina evidenciaram a implementação de políticas de saúde sexual, reprodutiva e de prevenção à aids entre mulheres profissionais do sexo tem mostrado eficácia, revelando que nas duas últimas décadas, houvera aumento do uso de preservativos entre elas (Madeiro \& Diniz, 2015).

Em estudo realizado em Guanambi-BA, verificou-se que o tempo de serviço se tornou um agravante para o uso do preservativo entre as mulheres profissionais do sexo, indicando que há correlação entre quanto mais tempo na profissão, menor é o uso da camisinha (Couto et al., 2019).

Embora o tempo de trabalho não tenha sido uma variável investigada neste estudo, estudos nacionais e internacionais apontam que a associação entre uso de preservativo e fatores socioeconômicos apresentam contradições, decorrentes, muitas vezes, do acesso às informações que elas têm sobre a prevenção do HIV (Shannon et al., 2015).

No âmbito político, atenta-se para a valorização da discussão sobre a importância do uso do preservativo, considerando seu papel, seus limites, estabelecer críticas dentro do contexto da prevenção do HIV/Aids. É necessário visar a diminuição da vulnerabilidade ao HIV/Aids nas populações de maiores riscos, especialmente, em MPS. Uma vez que estas pertencem a um grupo de pessoas mais vulneráveis à disseminação do vírus em virtude de sua atividade de risco. Além disso, possuem menor acesso aos meios de prevenção e serviços de saúde, em decorrência do preconceito e estigma por parte dos profissionais de saúde, e dificuldade em dialogar sobre o uso do preservativo (Sardinha; Santos \& Bonafé, 2015).

Com vistas a elucidação da maneira que as MPS se autopercebem vulneráveis ao HIV, essas foram questionadas acerca do fenômeno vulnerabilidade, bem como se já fizeram testes anti-HIV. Qual a frequência e que motivos as levaram ou levariam a fazê-lo, também foram objeto de interesse nesta investigação. Tais respostas estão apresentadas na Tabela 1. 
Tabela 1 - Caracterização da autopercepção de vulnerabilidade ao HIV entre mulheres profissionais do sexo. Floriano-PI, 2020. $(n=5)$.

\begin{tabular}{lll}
\hline Variáveis & N & $\%$ \\
Já fez o teste anti-HIV & 2 & 40,0 \\
\hline Sim, uma única vez & 3 & 60,0 \\
Sim, mais de uma vez & 0 & 0,0 \\
Não, nunca fez, mas faria & 0 & 0,0 \\
Não, e nunca faria & 1 & \\
Motivos que levam/levariam a fazer o teste anti-HIV & 20,0 \\
Curiosidade & 4 & 80,0 \\
Para descobrir, prevenir e tratar & 0 & 0,0 \\
Por preocupação com uma situação vivida & & \\
Não faria o teste por não querer saber o & 0 & 0,0 \\
resultado & & 80,0 \\
Sente-se vulnerável ao HIV & 0 & 20,0 \\
Sim & 4 & 0,0 \\
Não & 1 & \\
Não respondeu & & \\
\hline
\end{tabular}

Fonte: Autores.

Com relação ao teste anti-HIV, disponibilizado gratuitamente em Centros de Testagem e Aconselhamento - CTA pelo Sistema Único de Saúde - SUS (Brasil, 2018b), 60\% $(n=3)$ das mulheres relataram ter realizado o teste mais de uma vez, enquanto outras 40,0\% ( $\mathrm{n}=2)$, uma única vez.

Ao serem indagadas sobre os motivos pelos quais a levaram, ou ainda as levariam a realização do teste anti-HIV, 80,0\% ( $\mathrm{n}=4)$ referiu que realizaram e realizariam com objetivo de descobrir o resultado, prevenir-se de complicações, e em caso de infecção, tratar precocemente a síndrome, enquanto 20,0\% referiu encontrar motivação na curiosidade pelo resultado. Em se tratando de autopercepção de vulnerabilidade ao HIV, 80,0\% $(n=4)$ das mulheres participantes referiram não se sentirem vulneráveis, enquanto $20,0 \%(\mathrm{n}=1)$ não responderam.

Diante desse cenário, torna-se importante relatar que a busca pelo diagnóstico precoce do HIV, principalmente entre populações mais vulneráveis, representa um dos elementos mais importantes para o planejamento e execução da política de enfrentamento à aids no Brasil e no mundo (Who, 2015).

Embora notada a importância do interesse na detecção precoce de quaisquer infecções sexualmente transmissíveis, no Brasil, o diagnóstico do HIV ainda ocorre de maneira tardia. Dispensa-se especial atenção a essa informação, pois a aids constitui-se, a partir de um conjunto de tendências, sendo uma delas, a feminização e a concentração em populações específicas, como as de MPS. Considerando a existência de diferenças regionais, a prevalência nacional do HIV em profissionais do sexo gira em torno dos 4,8\%, enquanto entre mulheres, no geral, de 0,4\% (Szwarcwald et al., 2018).

Assim, existem recomendações nacionais e internacionais que preconizam que a inclusão de MPS no planejamento do controle ao HIV contribuem significativamente para a contenção da infecção pelo vírus na população, de maneira geral (Unaids, 2018 \& Who, 2015). 
Há necessidade de expansão do acesso a testes, de modo a evitar a precarização da detecção precoce e expandir os núcleos de assistência que ofereçam esse tipo de serviço, pois a priorização do diagnóstico precoce ocorre principalmente nas unidades básicas de saúde durante o período gestacional, o que isola características regionais e populações específicas, como é o caso das mulheres que vivem da prostituição (Sousa et al., 2017a).

\section{Conclusão}

A vulnerabilidade ao HIV/Aids é um aspecto de difícil compreensão. Quando analisada em dados quantitativos, oferece possibilidades limitadas de análise quando se consideram fenômenos tão complexos como a vulnerabilidade. Contudo, mesmo quando são utilizados dados qualitativos, as dificuldades na compreensão não desaparecem por completo.

Muitas são as razões pelas quais uma mulher profissional do sexo é vulnerável ao HIV/Aids. No que diz respeito à via de transmissão sexual, esta vulnerabilidade está diretamente relacionada ao não uso do preservativo em suas relações de trabalho. Ao investigar as percepções dessas mulheres no que tange ao uso do preservativo, foi identificada boa aceitação, especialmente ao não ter sido observada nenhuma indicação de desconforto, desprazer ou irritação com o seu uso.

A associação do seu uso como uma questão de saúde, que, citada por elas, está acima da oportunidade de ganhar dinheiro, configura-se como um fator de proteção. É indicador de sucesso de políticas públicas de enfrentamento da epidemia de aids no Brasil e no mundo, e de conscientização, especialmente, a públicos vulneráveis, dentre os quais estão inclusas as MPS.

A pesquisa demonstrou que as participantes possuíam algum conhecimento acerca do tema discutido, embora alguns equívocos tenham sido observados. Mesmo conhecendo algumas IST e suas formas de prevenção, as participantes ainda adotam comportamentos de risco, embora não se sintam vulneráveis.

Assim, identificados neste estudo os fatores que auxiliam na busca pela proteção sexual, em saúde e laboral dessas mulheres, é necessário que elas mereçam destaque nas ações de saúde. Aponta-se que capacitação profissional para o atendimento da demanda dessas profissionais, bem como campanhas de promoção ao uso de preservativos. Ressalta-se também, a necessidade de pesquisas de maior abrangência com esse público, devem ser rotina.

Ressalta-se também, a necessidade de pesquisas de maior abrangência com esse público, identificando fatores aqui não investigados, tais como o comportamento sexual dessas mulheres quando não estão a trabalho, para que sejam propostos mecanismos de defesa delas no contexto laborativo.

Cabe enfatizar que a análise do discurso do sujeito coletivo, método proposto para análise das percepções acerca da vulnerabilidade dessas mulheres, extraiu a unidade ideológica de um grupo de pessoas que trabalhavam muito próximas umas das outras, e não foi capaz de extrair discursos de mulheres que viviam em outras condições de trabalho, ambiente e realidade. Isso impossibilitou uma diversificação maior da amostra, sobretudo, quando da instalação da pandemia pela Severe acute respiratory syndrome coronavirus 2 - SARS-COV 2. A mesma implicou na suspensão da coleta de outros dados, por período indeterminado.

\section{Referências}

Alves, M. C., Jesus, J. P., \& Diaz, L. A. F. (2017). Autodeclaração da raça/cor no SUS: reflexões conceituais a partir da campanha realizada pelo estado do Rio Grande do Sul. Identidade! 22(1), 5-15.

Biernacki, P., \& Waldorf, D. (1981). Snowball Sampling: Problems and techniques of Chain Referral Sampling. Sociological Methods \& Research, 10(2), $141-163$.

Colin, E. C. D. S., \& Pelicioni, M. C. F. (2018). Territorialidade, desenvolvimento local e promoção da saúde: estudo de caso em uma vila histórica de Santo André, São Paulo. Saúde e Sociedade, 27, 1246-1260. 
Costa, F. M. et al. (2014). A percepção feminina quanto à vulnerabilidade de se contrair DST/aids. Revista da Universidade Vale do Rio Verde, 12(2), 880889.

Couto, P. L. S. et al. (2019). Correlação entre marcadores de vulnerabilidade social frente ao uso do preservativo por trabalhadoras sexuais. Saúde $e$ Pesquisa, 12(3), 591-599.

Cruz, N. L. et al. (2016). O cuidado com a saúde das mulheres profissionais do sexo: uma revisão narrativa. Disciplinarum Scientia Saúde, 17(3), 339-352.

Dourado, I. et al. (2015). Revisiting the use of condoms in Brazil. Revista Brasileira de Epidemiologia, 18, 63-88.

Fiorin, J. L. (2005). Elementos de análise do discurso. Editora Contexto.

Lima, I. M. B. et al. (2016). Modelo de decisão sobre o uso de preservativos: Uma regressão logística multinomial. Tempus Actas de Saúde Coletiva, 10(2), 67-80.

Madeiro, A. P., \& Diniz, D. (2015). Induced abortion among Brazilian female sex workers: a qualitative study. Ciência \& Saúde Coletiva, $20,587-593$.

Martins, T. A. et al. (2018). Incentivos e barreiras ao teste de HIV entre mulheres profissionais do sexo no Ceará. Revista de Saúde Pública, 52 , 64.

Ministério da Saúde. (2010). Diretrizes para organização e funcionamento dos CTA do Brasil. Brasília, DF: Ministério da Saúde.

Ministério da Saúde. (2018). Secretaria de Vigilância em Saúde. Departamento de DST, Aids e Hepatites Virais. Protocolo Clínico e Diretrizes Terapêuticas para Adultos Vivendo com HIV/Aids. Brasília, DF, 2018b. <http://www.aids.gov.br/pt-br/pub/2013/protocolo-clinico-e-diretrizes-terapeuticas-para-manejo-dainfeccao-pelo-hiv-em-adultos>.

Miranda-Ribeiro, P. et al. (2015). "É igual chupar bala com papel": a vulnerabilidade feminina ao HIV/Aids e o uso de camisinha em Belo Horizonte e Recife. E-book, 2, 391-416.

Nascimento, L. D. C. N. et al. (2018). Theoretical saturation in qualitative research: an experience report in interview with schoolchildren. Revista Brasileira de Enfermagem, 71(1), 228-233.

Patrício, A. C. F. A. et al. (2018). Análise de conceito da vulnerabilidade ao HIV/aids em mulheres profissionais do sexo. Revista Eletrônica de Enfermagem, 20,1-18.

Penha, J. C. et al. (2015). Fatores de risco para doenças sexualmente transmissíveis em profissionais do sexo do interior piauiense. Revista Gaúcha de Enfermagem, 36(2), 63-69.

Ribeiro, P. G. C. (2017). E aquela que costura pra fora?!: proposta de glossário para a tabuização e processo de formação de palavras para prostituta, no Maranhão. Dissertação (Programa de Pós-Graduação em Letras) - Universidade Federal do Maranhão, São Luís.

Santos, O. P. et al. (2019). Perfil sociodemográfico e avaliação do conhecimento das profissionais do sexo acerca das ISTs em um município na região metropolitana de Goiânia. Revista de Iniciação Científica e Extensão, 2(2), 81-88.

Sardinha, N. S., Santos, M. I. G., \& Bonafé, S. M. (2015). Estudo Observacional sobre HIV/AIDS em Indivíduos entre 13 e 19 Anos do Município de Maringá. Saúde e Pesquisa, 8(1), 71-78.

Shannon, K. et al. (2015). Global epidemiology of HIV among female sex workers: influence of structural determinants. The Lancet, $385(9962), 55-71$.

Soares, J. F. S. et al. (2015). A prostituição como profissão: uma análise sob a ótica das profissionais do sexo. Rev Saberes, 3(2), 63-75.

Sousa, R. M. R. B. et al. (2017a). Percepções de mulheres profissionais do sexo sobre acesso do teste HIV: incentivos e barreiras. Saúde em Debate, 41, 513525 .

Sousa, R. M. R. B. et al. (2017b). Prostituição, HIV/Aids e vulnerabilidades: a "cama da casa" e a "cama da rua". Cadernos Saúde Coletiva, 25(4), 423-428.

Szwarcwald, C. L. et al. (2018). Factors associated with HIV infection among female sex workers in Brazil. Medicine, 97(1 Suppl).

Unaids. (2018). Programa Conjunto das Nações Unidas sobre HIV/AIDS. Organização Mundial de Saúde. Estatísticas. <https://unaids.org.br/estatisticas/>.

Organização Mundial de Saúde. (2015). Diretrizes consolidadas sobre serviços de teste de HIV: 5Cs: consentimento, confidencialidade, aconselhamento, resultados corretos e conexão. 